Monatsschrift f. Geburtshülfe u. Gynäkologie 1920;51:I-IV

\title{
Contents, Vol. 51, 1920
}

\section{Inhalts - Verzeichnis}

Oríginalarbeiten.

\&gt; S Site

Albeek, V., Vorzeitige Lösung des normal befestigten Mutter-

kuchens 231

Brock, James, Die geriehtlich-mediziniscbie Bedeutung der

Sturzgeburten - . . 85

Buchacker, W., Krieg und Neugeborenenblennorrhoe . . . 406 Fink, Karl, L·uft- und Gasdepots am schwangeren und írisch

entbundenen Uterus als subakute Lebensgefahr und Todes-

ursache $38 \mathrm{~b}$ '

Fuhrmann, L., Zur 1OOjährigen Wiederkehr des Ritgenschen

.,Bauchscheidensehnittes" (Gastro-Elytrotomie) 1. Okt. 1020225 Gänssbauer, H., Multiple angeborene Cavernome .... 247 Grote, Rob., Die künstliche Frühgeburt beim engen Becken nach der Zangemeisterschen Methode 28

Hammerschlag, S., Über manuelle Plazentarlösung . . .311 Heymann, James, Ein Fall von

Spontangeburt eines reifen

Kindes in Stirnlage (Nachtrag zu dem Aufsatz unter diesem

Titel in Band 49, Heft 4 dieser Monatsschrift) . . . 303 II intzelmann, H., Chinin als Wehenmittel in der Abort-

behandlung .81

Hochenbichler, Ad., Beitrag zur Lehre von der Lösung

und Ausstoßung des Fruchtkuchens 1

.) aeger, Franz, Die rektale Untersuchung in der Geburtshülfe 160 Kayser, Konr. ,

Dermoidkystom als freie Geschwulst in der

Bauchhöhle 180

Kermauner, Fr., Zerreiñung der Nabelstrangvene .... 263 Köhler, Rob., Organextrakte als

Wehenmittel. Vorläufige

Mitteilung 240

K o $1 \mathrm{~d} \mathrm{e}, \mathrm{W}$. , Erwiderung an Weinbrenner ...... . 267

Koltonski, H., Über Osteomalazie 253

Krummacher, A., Styptysatum Burger bei profusen Menses.

II. Mitteilung .265

Kurtz, C., Alimentäre Amenorrhoe 367

Lietz, A., Über Diphtherie der Neugeborenen

Mayer, A., Über abnorme Kontraktionszustände der Harn

blase (Scheintumoren)

332

Muschallik, Ernst, Über die kombinierte intravenöse und

intramuskuLäre Injektion des Ghinins als Wehenmittel. . 378 
iv -

Scito

Quante, Über isolierte Koloparese auf entzündlich-reflektori-

scher Grundlage $\quad$ - 42

Roscher, H., Über einen Fall von Plazentartumor (Chorion-

angiom) und seine Stellung in der Onkologie325

Rubeli, Hektor, Struma lingualis und Schwangerschaft . 295 Samson, G., Ein kasuistischer

Beitrag zu den Entwieklungs-

störungen der Müllerschen Gänge 190

Scbmitt, Walther, Über Todesfcälle im Wochenbett an nieht-

puerperaler Sepsis 117

Schreiner, Ferd. , Über die Bedeutung der natürlicben Bakterizidie von Wundsekreten für die

Fruchtwasser-

bakterien 166

Sch war z, Botho, Zur Therapie der Stirnlagen 153

Schweitzer, Bernh., fiber hochsitzende retroperitoneale

Kystome . 171

Stix , Rud., Aktive oder konservative Behandlung des febrilen.

Abortus 394

Tschirde wahn , Hypoplasie und Tumor 186

Weinbrenner, C., Bemerkungen zu dem Aufsatz von Dr. W. Kolde: Die Behandlung der

Metropathia haemor-

rhagica und des Myoms mit Röntgenstrahlen266

- Erwiderung. Von W. Kolde 267

Wiegand, A., Statistisehes über Extrauteringravidität . . .316 Wortmann, W. , Die

Gesamtergebnisse der Behandlung des engen Beckens an der Bonner Frauenklinik in der Zeit

vom 1. April 1902 bis 1. April 1912 und vom 1. April 1912

bis 1. April 1919. 94

Zimmermann, Rob., Über einen Fall von Dicephalus

dibrachius dipus 194

Brief aus Amerika. Von Dr. Arthur Stein in Neuyork . . 124

Sammelreferat.

Bericht über die geburtshülfliche und gynäkologische Literatur

Dänemarksli'18-1919. Von Direktor Dr. Albeck 1/8Aarhus 414

Übersichts-Referat über 50 aus der Genfer Frauenklinik her-

vorgegangene Arbeiten von 1915-1918 48

Berichte.

Die 15. Tagung der üeutschen Gesellschaft für Gynäkologie in Berlin. Stimmungsbericht. Von Geh. Rat Prof. Seitz in Erlangen 133 Bericht über die Sitzungen. Von Dr. R. Hei nsius in Berlin 135

$-\mathrm{V}-$

Seite

Die 86. Versamnilung Deutscher Naturforscher und Ärzte in Nauheim. Stimmungsbei $\cdot$ icht. Von Prof. v. Jaschke in Gießen . 347 Bericht über die Sitzungen. Von Priv.-Doz. Dr. Dietrich in Göttingen 349 
Gustav Klein $\uparrow$. Yon Dr. Anton Hengge in München . .129 Friedrich Schatz $\uparrow$. Von Sañitätsrat Dr. Oldag in Mei\&en . 268

Tagesnachrichten und Personalien . . . 134, 207, 294, 348. 420

Vereins- und Literaturbeilage.

Gesellschaft für Geburtshilfe u. Gynäkologie zu Berlin. Sitzungen

vom 23. IV., 14. V. und 25. VI. 1920145

Sitzung vom 9. VII. $1920 \quad 216$

Gynäkologische Gesellschaft in Breslau. Sitzung vom 19.1.1920 208

Sitzung vom 24. II. $1920 \quad 271$

- Sitzung vom 23. III. 1920 . 352

- Sitzung vom 22. VI 421

Geburtshülflich·gynäkologische Gesellschaft von Mon†pellier . 148 Münchener gynäkologische Gesellschaft. Sitzung vom 14. III.

und 16. V. $1918 \quad 60$

Geburtshülflich·gynäkologische Gesellschaft in Wien. Sitzung

vom 10. und 24. Februar, 12. Mai 1920278

Referate aus geburtshülfhch-gynäkologischen Gesellschaften und

Vereinen 76, 217, 285, 427

Geburtshiilflich·gynäkologische Mitteilungen aus anderen medi $\cdot$

zinischen Gesellschaften und Vereinen 77,148, 218, 286, 363, 427

Buchbesprechungen 152, 219, 288

Literaturverzeich $\pi$ is $\quad 78,149,222,291,364,428$

Druckfehlerberichtigung 134, 294

Sachregister für Band LI-L1II 431

Namenregîster für Band LI-Mil 438 